\title{
THE SPLITTING OF THE HOCHSCHILD-SERRE SPECTRAL SEQUENCE FOR A PRODUCT OF GROUPS
}

\author{
BY
}

\author{
UWE JANNSEN
}

\begin{abstract}
We show that the Hochschild-Serre spectral sequence for a product $G \times H$ of abstract or profinite groups splits for $G$-modules, and that this splitting is not functorial.
\end{abstract}

In an unpublished preprint [2] from 1977, Milgram uses a certain "Künneth formula" for a product of profinite groups. This formula is quite useful, but there seems to be no proof in the literature. Since it is not clear how to deduce it from the usual Künneth relations, and since the mere statement contains the danger of a misinterpretation, it is the aim of this note to outline a proof and to give an example against a possible misuse.

THEOREM. Let $G$ and $H$ be profinite groups, and let $B$ be a discrete $H$-module, regarded as a $(G \times H)$-module via trivial action of $G$.

(a) The Hochschild-Serre spectral sequence

$$
E_{2}^{p, q}=H^{p}\left(G, H^{q}(H, B)\right) \Rightarrow H^{p+q}(G \times H, B)
$$

degenerates, and splits in the sense that there is a decomposition

$$
H^{n}(G \times H, B) \cong \bigoplus_{p+q=n} H^{p}\left(G, H^{q}(H, B)\right)
$$

(b) The last decomposition is non-canonical, in particular it cannot be made functorial in B. However, for a fixed $B$ it can be made functorial in $G$.

Proof. (a) There is a $\mathbb{Z}$-flat, $G$-acyclic resolution of $\mathbb{Z}$, for example the complex $D^{\cdot}(G, \mathbb{Z})$ of not necessarily covariant continuous cochains with values in $\mathbb{Z}$, for which $D^{\cdot}(G, \mathbb{Z})^{G}=C^{\cdot}(G, \mathbb{Z})$, the usual complex of continuous cochains ([3]I2.2). If $I^{\cdot}$ is any $H$-acyclic resolution of $B$, then $D^{\cdot}(G, \mathbb{Z}) \otimes I$ is a $(G \times H)$-acyclic resolution of $\mathbb{Z} \otimes B=B$. On the other hand, let $\tau_{\leqq m}$ be the canonical filtration of $K^{\cdot}=I^{\cdot H}$

Received by the editors September 20, 1988.

Research supported by DFG and MSRI, Berkeley.

AMS (1980) Classification: Primary 20J05, Secondary 18G15, 20E18.

(c) Canadian Mathematical Society 1988. 


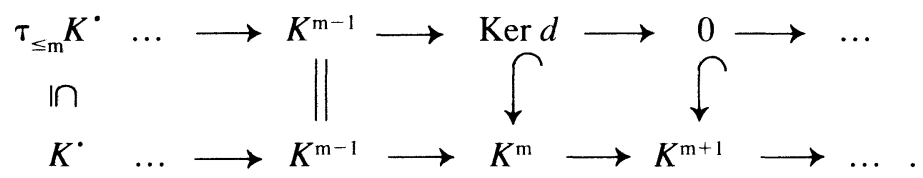

Then by the flatness of $D^{\cdot}(G, \mathbb{Z})$ the complex $D^{\prime}(G, \mathbb{Z}) \otimes K^{\prime}$ with the induced filtration is a filtered $G$-acyclic resolution of $\left(K, \tau_{\leqq m}\right)$. We deduce that the cohomology of

$$
\left(D^{\cdot}(G, \mathbb{Z}) \otimes I^{\cdot}\right)^{G \times H}=\left(D^{\cdot}(G, \mathbb{Z}) \otimes K^{\cdot}\right)^{G}=C^{\cdot}(G, \mathbb{Z}) \otimes K^{\prime}
$$

is the $(G \times H)$-cohomology of $B$, and that the Hochschild-Serre spectral sequence is associated to the filtration induced on it by $\tau_{\leqq m}$ (cf. [1]1.4). Hence it suffices to prove that the maps

$$
H^{n}\left(C^{\cdot}(G, \mathbb{Z}) \otimes \tau_{\leqq m} K^{*}\right) \rightarrow H^{n}\left(C \cdot(G, \mathbb{Z}) \otimes \tau_{\leqq m+1} K^{*}\right)
$$

have left inverses for all $m$ and $n$. By the flatness of $C^{\cdot}(G, \mathbb{Z})$ we may replace $\tau_{\leqq m+1} K^{\cdot}$ by any quasi-isomorphic complex, so we may assume that $\tau_{\leqq m+1} K^{\cdot}$ has $\mathbb{Z}$-free com-

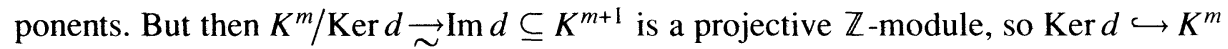
and, consequently, $\tau_{\leqq m} K^{\cdot} \rightarrow \tau_{\leqq m+1} K^{\prime}$ has a left inverse, which gives the desired result.

(b) Since the spectral sequence is functorial, and the splitting above only depends on $C^{\cdot}(H, B)$, the functoriality in $G$ is clear. For the functoriality in $B$ look at the following counter-example. Let $G$ and $H$ be cyclic groups of order $p, p$ a prime number. If $B=\mathbb{Z} / p^{3}$ with a generator of $H$ acting as multiplication by $1+p^{2}$, we have an exact sequence of $(G \times H)$-modules

$$
O \rightarrow \mathbb{Z} / p \stackrel{\alpha}{\rightarrow} B \rightarrow \mathbb{Z} / p^{2} \rightarrow O
$$

with trivial action on kernel and cokernel. From the long exact sequence

$$
\begin{array}{cc}
\ldots \longrightarrow H^{1}\left(G \times H, \mathbb{Z} / p^{2}\right) & \longrightarrow H^{2}(G \times H, \mathbb{Z} / p) \stackrel{\alpha_{*}}{\longrightarrow} H^{2}(\mathrm{G} \times H, B) \longrightarrow \ldots \\
R & R \\
(\mathbb{Z} / p)^{2} & (\mathbb{Z} / p)^{3}
\end{array}
$$

we conclude that the map $\alpha_{*}$ induced by $\alpha$ cannot be zero, although it is zero on all $E_{2}^{p, q}$ for $p+q=2$. Namely, $B$ is cohomologically trivial for $H$, so $H^{1}\left(G, H^{1}(H, B)\right)=$ $0=H^{0}\left(G, H^{2}(H, B)\right)$, and it is easy to see that the map

$$
H^{2}(G, \mathbb{Z} / p)=H^{2}\left(G, H^{0}(H, \mathbb{Z} / p)\right) \stackrel{\alpha_{*}}{\longrightarrow} H^{2}\left(G, H^{0}(H, B)\right)=H^{2}\left(G, \mathbb{Z} / p^{2}\right)
$$

is zero.

Remark. The same proof shows the following. If $R$ is a Dedekind ring, $B$ is a discrete $R[H]$-module, and $A$ is a discrete $R[G]$-module, which is flat as an $R$-module, then there is a (non-canonical) decomposition

$$
\left.H^{n}\left(G \times H, A \otimes_{R} B\right) \cong \bigoplus_{p+q=n} H^{p}(G, A) \otimes_{R} H^{q}(H, B)\right) .
$$


Also, everything remains true for discrete groups.

ACKNOWLEDGEMENT. I thank C. Riehm for calling my attention to this problem, and the McMaster University for its hospitality during my stay.

\section{REFERENCES}

1. P. Deligne, Théorie de Hodge II, Publ. Math. I.H.E.S. 40 (1971), 5-58.

2. R. J. Milgram, The Determination of the Schur Subgroup, preprint 1977.

3. J. P. Serre, Cohomologie Galoisienne, Springer Lecture Notes in Math. 5 (1973).

Fakultät für Mathematik

Universität Regensburg

Universitätsstraße 31

D-8400 Regensburg 